\title{
Effect of backward injection with combined hole on film colling performance
}

\author{
F. Ben Ali Kouchih, K. Boualem and A. Azzi \\ Laboratoire d'aéro-hydrodynamique navale, Département de Génie Maritime, Université des Science et de la Technologie d'Oran Mohamed \\ Boudiaf, USTO-MB, Oran, 31000 Algérie \\ Phone: +213077914896
}

\begin{abstract}
This study investigates the film cooling performance and flow features of backward injection with combined hole. This concept is evaluated by comparison to forward injection with combined hole and other forms with both injections, forward and backward. The shapes are namely, cylindrical hole, conical hole and fan-shaped hole. The eight configurations are computed for three blowing ratios $M=0.5,1.0$ and 1.5. The air coolant was injected through holes inclined at $35^{\circ}$ and $155^{\circ}$ for forward and backward injection respectively. The lateral averaged film cooling effectiveness and the distribution of adiabatic film cooling efficiency are studied using commercial software ANSYS- CFX. In addition, several velocity vectors and contours are presented for analyzing the thermal behavior. The results show that a uniform coverage is obtained by the backward injection which leads to best cooling. The maximum improvement of film cooling is obtained by backward injection with combined hole at $\mathrm{M}=1.5$.
\end{abstract}

ARTICLE HISTORY

Received: 26 $6^{\text {th }}$ Apr 2020

Revised: $20^{\text {th }}$ Jan 2021

Accepted: 25 $5^{\text {th }}$ Feb 2021

\section{KEYWORDS}

Film cooling effectiveness; forward injection; backward injection; fan-shaped hole; combined hole.

\section{INTRODUCTION}

Increasing the efficiency of turbines operating on a recovered Brayton cycle involves increasing the inlet temperature of the turbine. However, the metal of blades must be massively cooled in order to resist temperatures exceeding $1600 \mathrm{~K}$ [1]. According to these conditions, a variety of cooling techniques of turbine blades has been tested and employed. Air film cooling is the most common and efficient system used in the industry for cooling gas turbine blades. The coolant air is taken from the compressor and injected through single or multiple rows of holes forming a cooler film over the blade surface to reduce the heat transfer. Forward injection through cylindrical orifices is the most used technique in film cooling. The most common disadvantage of this system is the flow takes off from the surface in the form of two vortices. The counter-rotating vortices (CRVP) bring the hot gases into the jet and destroy the cold air protective film. Several forms have been used to improve the film cooling performance. Goldstein et al [2,3] conducted research on the effect of film cooling hole geometry on film cooling performance. Their experimental results show that the improvement of the film cooling efficiency is achieved by the use of holes having lateral fan diffusion. Gritsh et al [4] investigated three kinds of film cooling holes, cylindrical holes, fan-shaped holes and laidback fan-shaped holes, taking into account the effect of Mach number and jet orientation on the flow field. They found that the laidback fan-shaped holes perform better than other holes. Saumweber et al [5] conducted an experiment to study the

effect of free-stream turbulence on the performance of four jet shapes, cylindrical holes, holes with expanded exits, a fan-shaped, and a laidback fan-shaped hole. The findings showed that the effect of the turbulence intensity was much more pronounced for the shaped holes. Lee et al [6] utilized transient liquid crystal thermograph to measure the film cooling efficiency of a discrete hole and a forward expanded hole with $\beta=0^{\circ}, 45^{\circ}$ and $90^{\circ}$ embedded in a concave surface. Their results show that the injection using a forward expanded hole yields higher effectiveness and lower heat transfer than a simple angle hole. The study conducted by Miao and Wu [7], compare the efficiency of three different hole designs, cylindrical hole, forward diffused hole and laterally diffused hole varying the blowing ratio from 0.3 to 1.5 . Their main finding is that the laterally diffused configuration improves the spanwise-averaged film cooling effectiveness.

Azzi and Jubran [8] have studied numerically the configuration 'console' to control the intensity of counter-rotating vortex pair (CRVP). They showed that the new geometry offers a flow structure without the two counter-rotating vortices responsible for the degradation of the protective film. In the same context, khorsi and Azzi [9] indicated that the converging slot hole (Console) gives better efficiency than the classical cylinder hole. The results Guangchao et al [10] showed that the film cooling effectiveness significantly increases and the heat transfer decreases by increasing the momentum flux ratio for the injection through fanned holes. Liu et al [11] employed the transient liquid crystal technique to measure the film cooling efficiency, heat flux ratio and discharge coefficient of Console jet with different exit-entry area ratios. They found that the Console hole with smaller area ratio provides the best thermal protection. In other study, Liu et al [12] presented a measurement of film cooling performance of waist-shaped slot hole, which was compared to two types of console holes. The best thermal protection was obtained by console holes with small area ratio.

Nguyen et al [13] presented two types of conical-shaped holes and compared to the traditional cylindrical hole. The experimental and numerical results showed that the conical shaped holes perform better for the low and medium blowing ratios by keeping the flow attached to the surface. Chang et al [14] analyzed the flow structure and film cooling mechanism 
of a combined-hole. Their results showed that this design can eliminate the kidney vortices that are harmful for film cooling. Hassan et al [15] have combined two cylindrical holes as a unit to enhance the film cooling performance at $\mathrm{M}=$ 0.5 and $\mathrm{M}=0.75$. A novel combined hole was published by Wang et al [16]. The efficiency of this design to improve the film cooling effectiveness and reduction the aerodynamic loss is compared to conical and cylinder hole at $\mathrm{M}=0.25,0.5$, 0.75 , and 1.0. They reported that the combined hole provides uniform film cooling performance in both downstream and lateral directions.

In order to eliminate CRVP or reduce their size, $\mathrm{Na}$ and Shih [17] have placed a ramp upstream of a coolant hole; the results show that the size of counter-rotating vortex pair can be controlled, which leads to improve the cooling performance. Zaman et al [18] and Shinn et al [19] have presented the distributions of film cooling behind micro-ramp which generate anti-vortexes that can delay the jet lifting off. An et al [20] conducted an experiment to study the effect of a short crescent-shaped block placed at the downstream of a cylindrical cooling hole on the cooling performance. The findings indicated that the presence of this cresent-shaped block increase the lateral averaged film cooling. The geometry optimization of crescent-shaped block was studied by Zhang and Wang [21]. They found that each blowing ratio has an optimal block. Zhou and $\mathrm{Hu}[22,23]$ inspired from the shape of sand dunes, a new ramp design (Barchan-duneshaped).Their results indicate that this new concept affects the CRVP, and enhance the film cooling efficiency with lower aerodynamic losses.

In recent years, other researchers have developed film cooling systems, among them, Li [24] who used backward injection holes over a flat plate, where the coolant air was injected in the opposite direction to that of the main stream. He presented detailed flow structure and thermal field of the backward cooling. The results showed that the backward hole (BH) can improve the film cooling effectiveness with a larger pressure loss penalty. Subbuswamy et al [25] have improved the film cooling effectiveness using backward injection with fan-shaped holes. Park et al. [26] combined the forward and backward injection together taking into account the effect of blowing ratios (0.5 to 2.0), the density ratio was set to the unity. They found that this configuration can enhance the effectiveness of cooling laterally due to the dispersion of the backward injection. Singh et al [27] reported in their study that the discharge coefficient decreases by the backward injection compared to the forward injection, in particular at high blow ratio (1.0 to 3.0). Zhao et al [28] examined backward injection with a ramp placed upstream of a coolant hole. The coolant flow generated by the upstream ramp and downward vortex by the backward hole can suppress the intensity the kidney vortexes, which leads to improve the film cooling effectiveness laterally.

Researchers mentioned above made various attempts to improve the film cooling performance. The aim of the present study is to investigate film cooling performance on flat plate based on many hole configurations, i.e., cylindrical hole, conical hole, fan-shaped hole, and combined hole with forward and backward injection. The eight designs are analyzed at three blowing ratios 0.5, 1.0 and 1.5. The obtained results are compared with the experimental data of Sinha et al [29].

\section{FLOW GEOMETRY AND BOUNDARY CONDITIONS}

\section{Flow Geometry}

In the present investigation, the computational domain for numerical simulations is referred to the experimental work of Sinha et al [29], consists of a flat plate provided with an injection hole of diameter D $=12.7$ mm with length to diameter rate $\mathrm{L} / \mathrm{D}=1.75$, and inclination angle of $35^{\circ}$. Figure 1 presents a detailed description of the calculation domain that extends from the input plane up to 49D in streamwise direction of flow and from the flat plate to 10D in the vertical direction. The Plenum dimensions are (8D x 4D x 3D).

Figure 2 and Table 1 show the four-hole shapes used in the present study, cylindrical hole, conical hole, fan-shaped hole, and combined hole. All forms are examined in both directions, forward and backward injections. The cylindrical hole configuration is similar to the experimental work of Sinha et al [29]. The dimensions of the conical hole, fan-shaped hole and combined hole were taken from the numerical study of Wang et al [16]. In the conical hole case, a cylindrical shape of the coolant jet changes to a conical shape at $\mathrm{x}=1.75 \mathrm{D}$. While, the fan-shaped hole has a lateral diffusion angle of $37^{\circ}$ at $x / D=1.75$. As for, the combined hole configuration is produced by a combination of three cylindrical holes with the same diameter, which two side holes were added to the main hole by an angle of $30^{\circ}$ in a lateral diffusion.

\section{Boundary Condition}

The same boundary conditions are applied for all test holes. The main flow velocity and temperature were specified at the inlet, $\mathrm{U}_{\infty}=20 \mathrm{~m} / \mathrm{s}$ and $\mathrm{T}_{\infty}=300 \mathrm{~K}$. For the coolant condition, the temperature was set to $T_{c}=250 \mathrm{~K}$ and three injection ratios of $0.5,1.0$ and 1.5 are considered, $M=\left(\rho_{c} U_{c} / \rho_{\infty} U_{\infty}\right)=0.5,1.0$ and 1.5. Where $\rho_{c} U_{c}$ are the density and velocity of the coolant jet, and $\rho_{\infty} \mathrm{U}_{\infty}$ are for the main stream. The density ratio between the injection flow and the main flows is kept to unit.

A pressure of 1 bar is considered at the outlet ant the top boundary of the domain. In this investigation, the temperature is considered as a passive scalar, an arbitrary difference of $50^{\circ} \mathrm{C}$ is set between the mainstream and coolant air. For comparison purpose, a non-dimensional temperature is defined by Eq. (1) and called adiabatic film cooling effectiveness. It varies between one and zero. For further discussions, a laterally averaged adiabatic film cooling effectiveness is defined by Eq. (2) and gives a wide view of the cooling process in the spanwise direction where $\mathrm{x}$ was varied between 0 and $30 \mathrm{D}$. 


$$
\eta=\left(T_{\infty}-T_{a w}\right) /\left(T_{\infty}-T_{c}\right)
$$

Where $\mathrm{T}_{\infty}$ is hot air temperature, $\mathrm{T}_{\mathrm{aw}}$ is the adiabatic wall temperature and $\mathrm{T}_{\mathrm{c}}$ is the coolant temperature.

$$
\bar{\eta}=1 / L \int_{0}^{l} \eta d z
$$

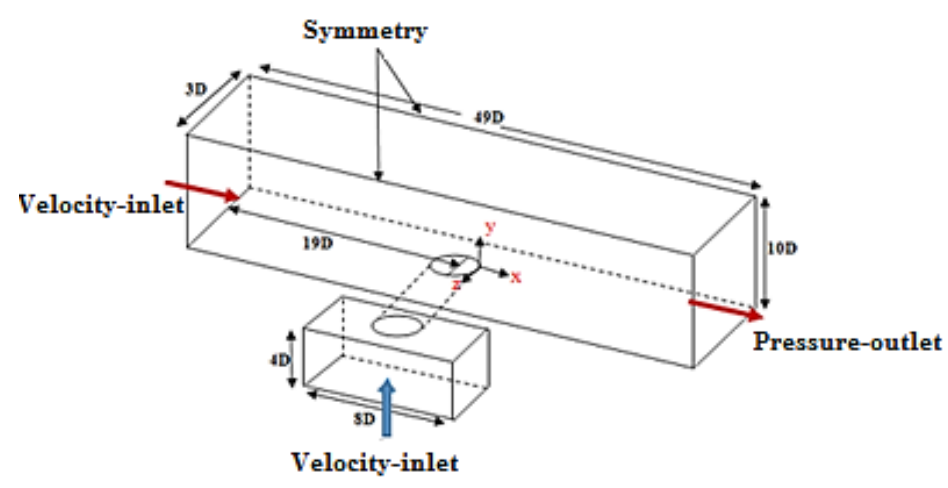

(a)

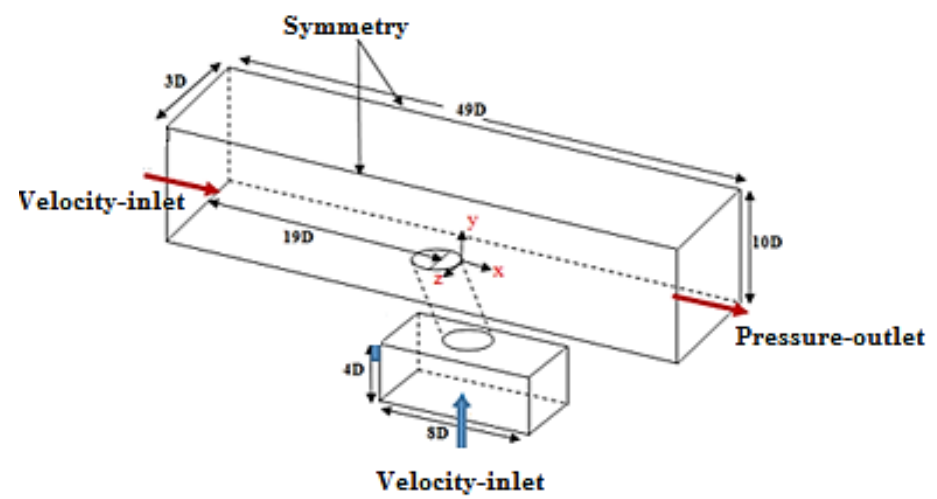

(b)

Figure 1. Computational domain: (a) film cooling with forward injection and (b) film cooling with backward injection
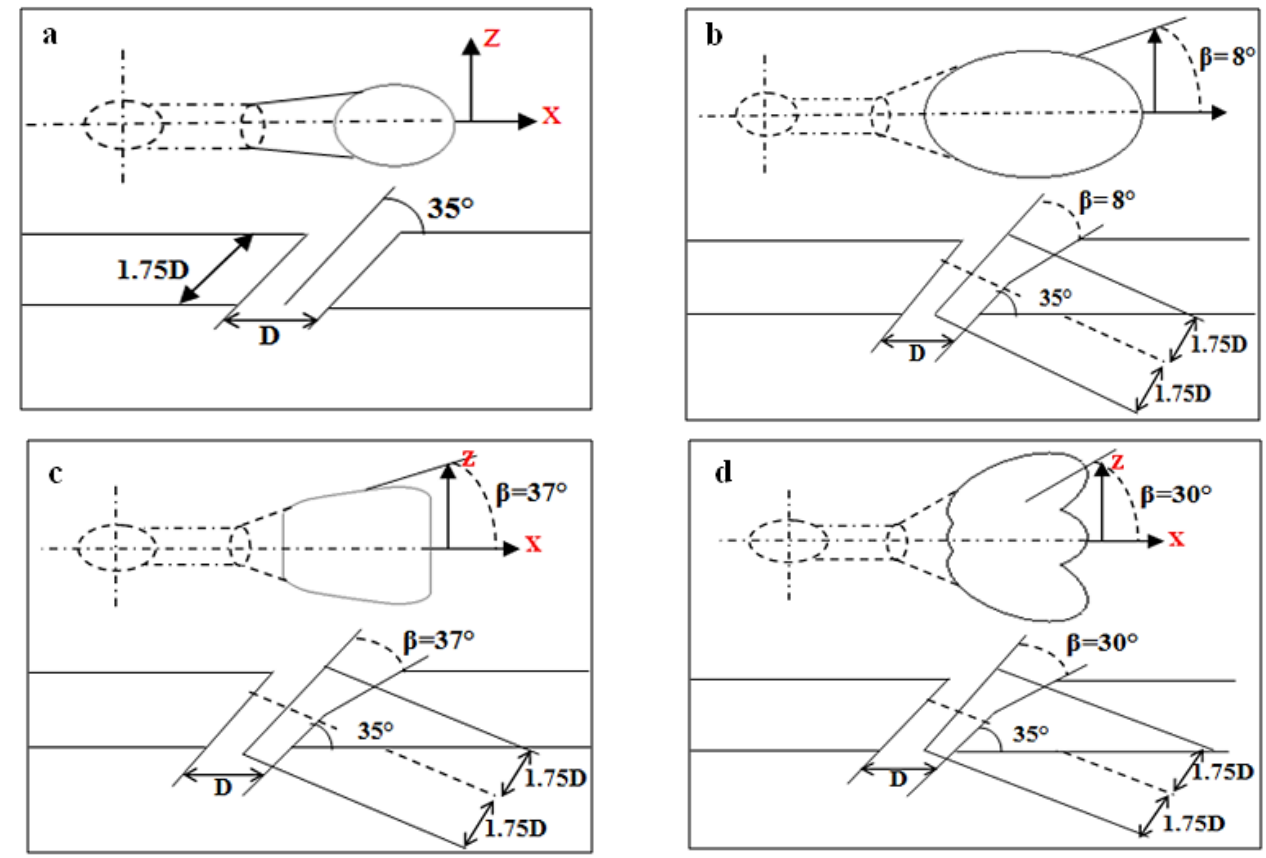

Figure 2. Sketch map of film cooling holes: (a) Cylindrical hole, (b) conical hole and (c) fan-shaped hole and (d) combined hole 
Table 1. Holes cases

$\begin{array}{ll}\text { Case 1: } & \text { Cylindrical hole with forward injection }(\mathrm{Cyl}-\mathrm{FH}) \\ \text { Case 2: } & \text { Cylindrical hole with backward injection }(\mathrm{Cyl}-\mathrm{BH}) \\ \text { Case 3: } & \text { Conical hole with forward injection }(\mathrm{Con}-\mathrm{FH}) \\ \text { Case 4: } & \text { Conical hole with backward injection }(\mathrm{Con}-\mathrm{BH}) \\ \text { Case 5: } & \text { Fan-shaped hole with forward injection }(\mathrm{F}-\mathrm{SH}-\mathrm{FH}) \\ \text { Case 6: } & \text { Fan-shaped hole with backward injection }(\mathrm{F}-\mathrm{SH}-\mathrm{BH}) \\ \text { Case 7: } & \text { Combined hole with forward injection }(\mathrm{Com}-\mathrm{FH}) \\ \text { Case 8: } & \text { Combined hole with backward injection }(\mathrm{Com}-\mathrm{BH})\end{array}$

\section{NUMERICAL MODEL AND GRID INDEPENDENCE}

The numerical model is composed by the Reynolds Averaged Navier-Stokes Equations (RANS), a turbulence closure model and the temperature equation. The system is resolved by use of the Finite Volume method integrated to the ANSYSCFX commercial software. According to numerous previously published works [30], the RNG k-epsilon turbulence model seems to be well adapted to film cooling computations and gives valuable results taking into account the available computational facilities. The second order upwind scheme is used for the spatial discretization of convective terms and the SIMPLEC algorithm for pressure-velocity coupling.

In order to eliminate the interpolation errors, three computational grids are generated with the ICEM grid generator. The three grids are composed by approximately 1, 1.5 and 2 million hexahedral elements respectively. The computational elements are arranged in a multi-bloc strategy and adopt the O-grid structure in the vicinity of the hole injection. Figure 3 shows some details of the grid arrangement.

The centerline adiabatic film cooling effectiveness which located in the horizontal line passing through the geometrical center of the orifice compared with available experimental data is illustrated in figure 4 . The trend is well reproduced by all computations and presents minimal changes when moving from one grid to another. According to these results, the grid of 1,500,000 elements is adopted for all computations.

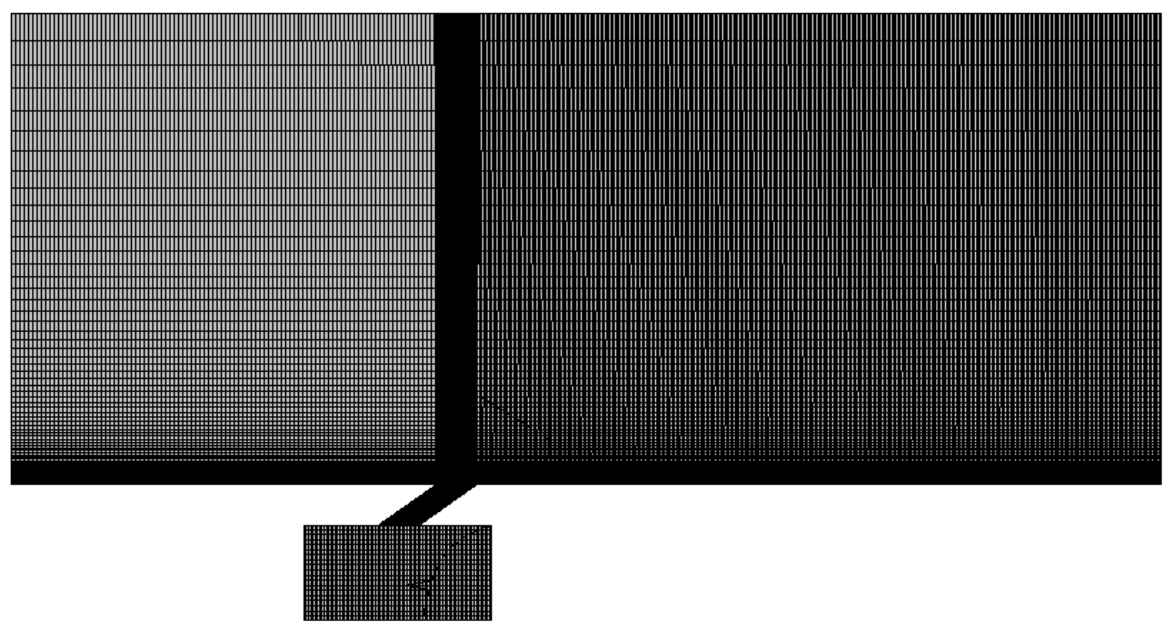

(a)

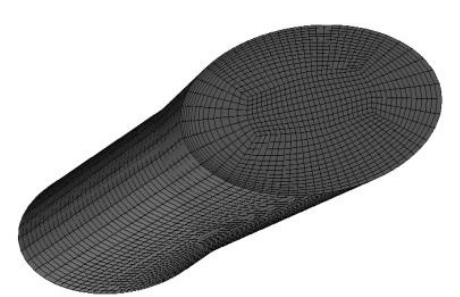

(b)

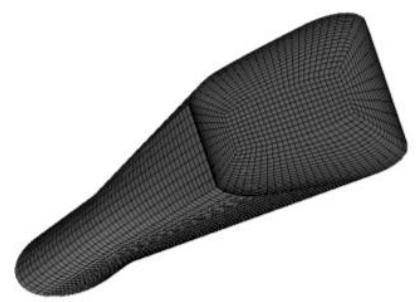

(c)

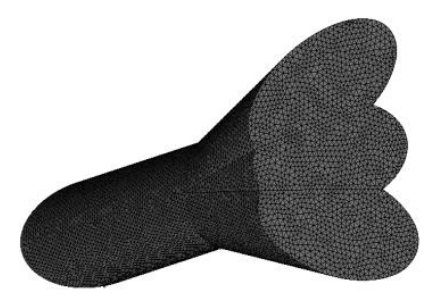

(d)

Figure 3. (a) The calculation grid, (b) conical hole, (c) fan-shaped hole and (d) combined hole 


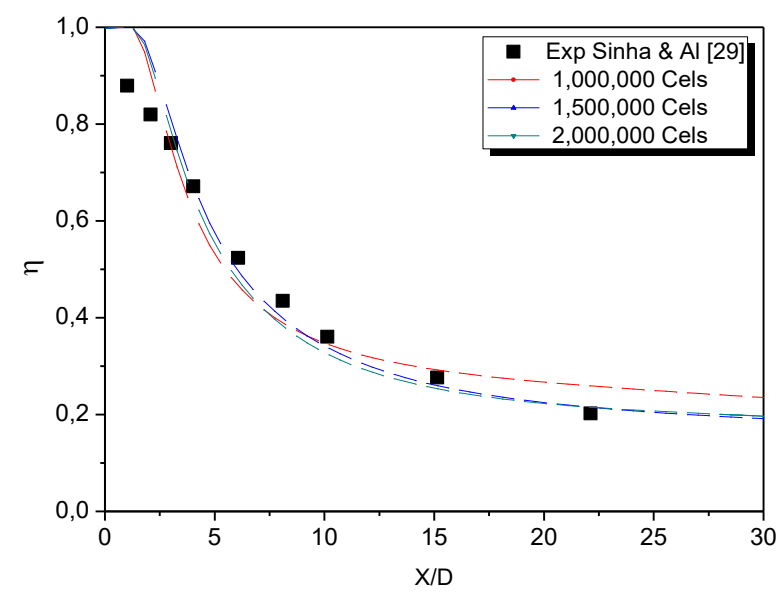

Figure 4. Mesh independence validation

\section{RESULTS}

\section{Film Cooling Effectiveness}

The centreline effectiveness and the laterally averaged cooling efficiency profiles for the twenty-four cases are plotted in Figure 5 and 6, respectively. It can be clearly seen that for all configurations, the film cooling efficiency (the centerline and laterally averaged) increases near the jet outlet and decrease along (X/D). The analysis of Figures 5 and 6 indicate that the shape and orientation of the jet have a significant effect on the distribution of cold air.

For Cyl-BH configuration, it can be clearly seen that for all blowing ratios the film cooling effectiveness increases near the jet outlet and decrease along (X/D). Whereas, a jump up is observed in the backward film hole case at M=1.0 and 1.5 which creating due to the detachment and re-attachment of the coolant flow. The cold air is well distributed in the centerline and the lateral compared with the Cyl-FH, this is doing by the reverse injection that helped the jet to reattach on the flat plate and cover the whole surface.

The Con-BH geometry at $\mathrm{M}=1.0$ and 1.5 shows an improvement in laterally averaged film cooling effectiveness. While Con-FH is more effective in the centreline at low blowing ratio $(\mathrm{M}=0.5)$, on the contrary, at a high blowing, it loses its efficiency. By applying the forward injection with fan-shaped hole and combined hole at $M \leq 1$, an increase in centerline and lateral averaged film cooling efficiency can be observed compared with $(\mathrm{F}-\mathrm{SH}-\mathrm{BH})$ and $(\mathrm{Com}-\mathrm{BH})$.

It is interesting to note that at high blowing ratios $(\mathrm{M}=1.5)$, for all the jet forms, the use of the backward injection yields an enhancement in the laterally averaged film cooling efficiency.
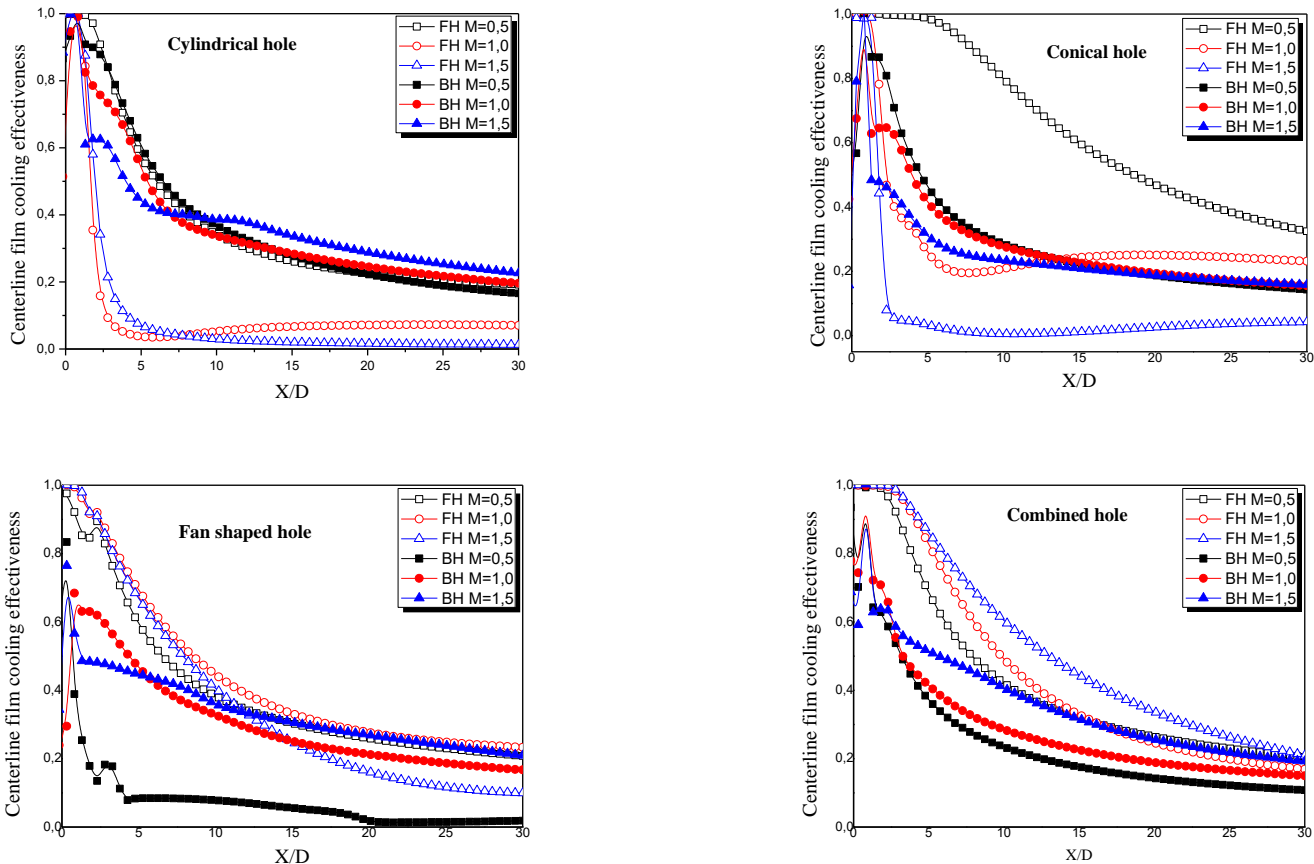

Figure 5. Comparison of Centerline film cooling effectiveness for $\mathrm{M}=0.5,1.0$ and 1.5 

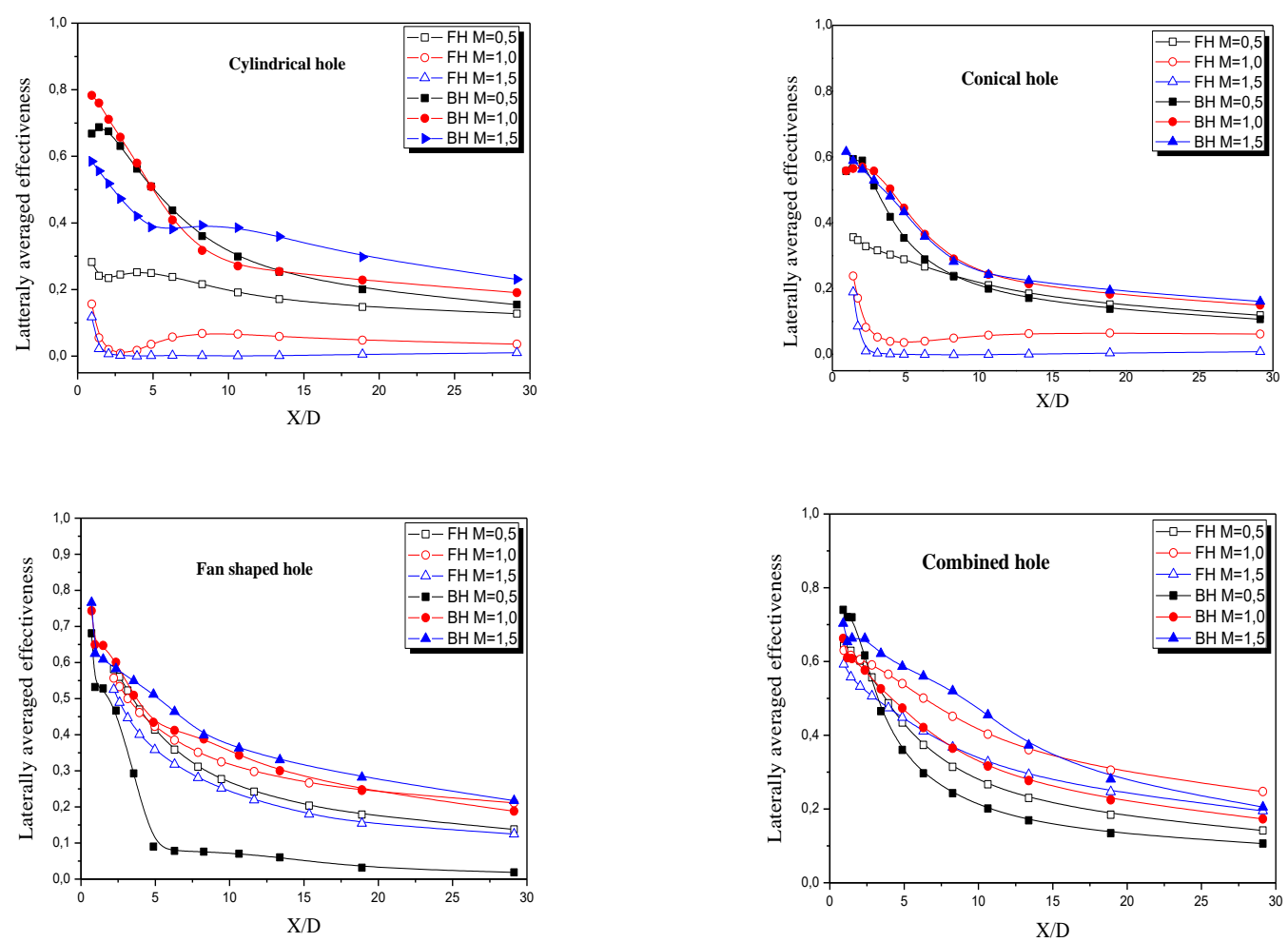

Figure 6. Comparison of lateral averaged film cooling effectiveness for $\mathrm{M}=0.5,1.0$ and 1.5

\section{Area Weighted Film Cooling Effectiveness}

In order to evaluate the film cooling performance overall the flat plate, the area weighted average film cooling effectiveness for all considered configurations is presented in figure 7. The area weighted average film cooling effectiveness is a simple scalar calculated by the post-processor module of the software, that it is performed for all the flat plate $(0<\mathrm{x} / \mathrm{D}<30)$.

According to figure 7 and table 2, the cylindrical hole with backward injection (Cyl-BH) provides a highest value of effectiveness at all blowing ratios compared with the Cyl-FH. It illustrates that the conical hole is more efficiency with backward injection than forward injection especially at higher blowing ratios. At M=1 and 1.5, the Con-BH configuration can produce much more uniform cooling coverage as compared to Con-FH. Area weighted average film cooling effectiveness for F-SH-BH and Com-BH are almost similar at $\mathrm{M}=1.5$, it can reach to 0.241 . This indicates the reattachment of the cooling jet to the flat plate, which is well cooled. While, at lowest blowing ratio $\mathrm{M}=0.5$ the F-SH-FH and Com-BH cases present higher area weighted average effectiveness values.

Table 2. Data of area weighted effectiveness

\begin{tabular}{ccccccc}
\hline & FH M0.5 & BH M0.5 & FH M1.0 & BH M1.0 & FH M1.5 & BH M1.5 \\
\hline Cylindrical hole & 0.108 & 0.18 & 0.03 & 0.202 & 0.012 & 0.161 \\
Conical hole & 0.127 & 0.133 & 0.04 & 0.163 & 0.02 & 0.178 \\
Fan shaped hole & 0.143 & 0.108 & 0.175 & 0.208 & 0.126 & 0.235 \\
Combined hole & 0.164 & 0.138 & 0.232 & 0.189 & 0.19 & 0.241 \\
\hline
\end{tabular}




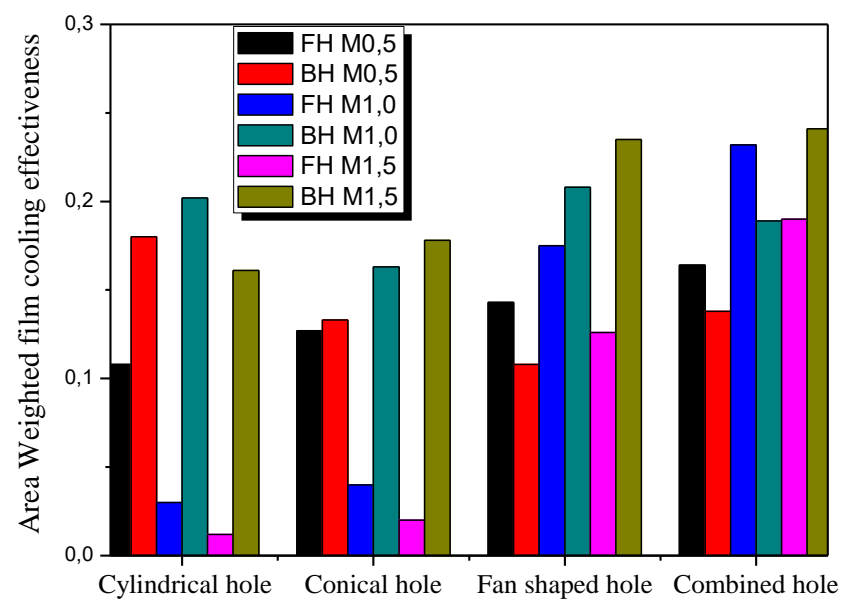

Figure 7. Area weighted average film cooling effectiveness for all hole configurations at all blowing ratios

\section{Thermal Field and Vortex Structure}

Figure 8 shows a comparison of the wall film cooling effectiveness for each configuration with forward and backward injection at $\mathrm{M}=0.5$ and $\mathrm{M}=1.0$. Increasing the blowing ratio in the Cyl-FH case reduces the cooling efficiency due to the coolant flow detachment from the surface; contrary to the Cyl-BH design that keeps the flow attached to the flat plate. For the Con-FH, F-SH- FH and Com-FH designs, the level of the film cooling effectiveness near the outlet jet is high but is not evenly distributed on the plate. The use of backward injection with combined hole (Com-BH) can significantly improve the downstream and the lateral film cooling effectiveness leading to uniform distribution. This is due by the jet direction from the backward hole where the mainstream collided with secondary flow and bended it in the direction of the flow, which can eliminate the flow detachment leading to more effective protection of the wall from hot mainstream.

One of the main challenges associated with the use of film cooling is the formation of counter rotating vortices (CRVP) that recall the hot gases above the jet and destroy the cold air protective film. The counter rotating vortexes pair (CRVP) and temperature distributions on the $y-z$ plane at $x / D=3, x / D=6$ and $x / D=10$ are displayed in figure 9. Each line shows one of the hole design tested at $M=1.0$. For the forward injection, the interaction between the coolant air and the mainstream generates a vortex pair near the film hole which prevents the air to be distributed properly. As shown in temperature contours and velocity vectors, smaller Anti-vortexes were generated in the fan-shaped and combined holes cases. The presence of these Anti-vortexes suppresses the detachment of the jet and diminishes adequately the strength of the vortex pair, which is presented clearly in the cylindrical case. In contrast, this vortex pair disappears in the backward case for all hole configurations. This causes that the backward injection has a larger effect on the structure size and strength of CRVP.

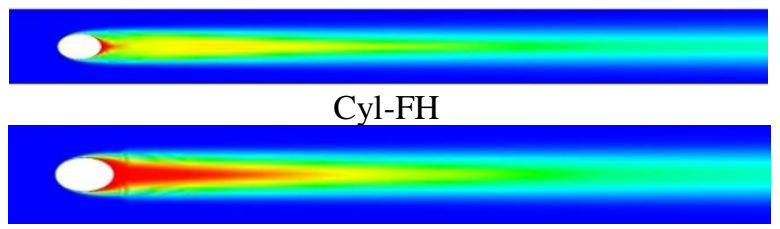

Con-FH

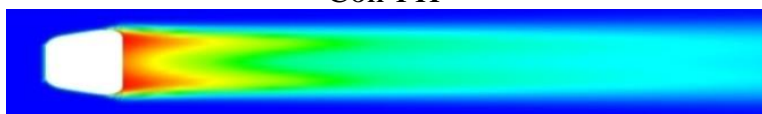

F-SH- FH

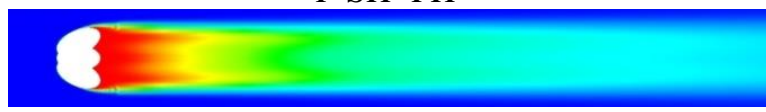

Com-FH

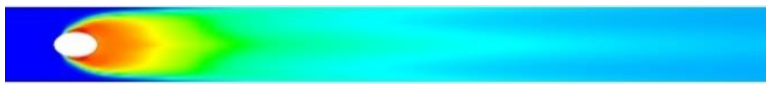

Cyl-BH

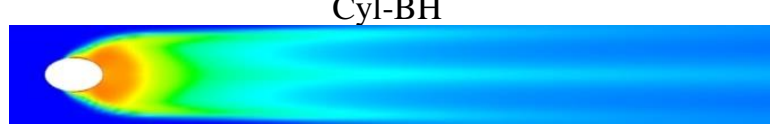

Con- BH

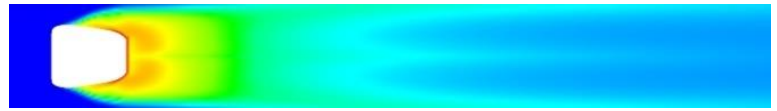

F-SH- BH

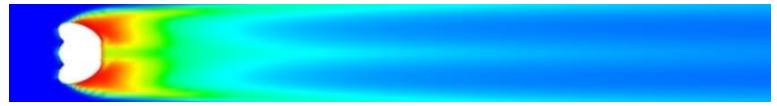

Com-BH

$M=0.5$ 


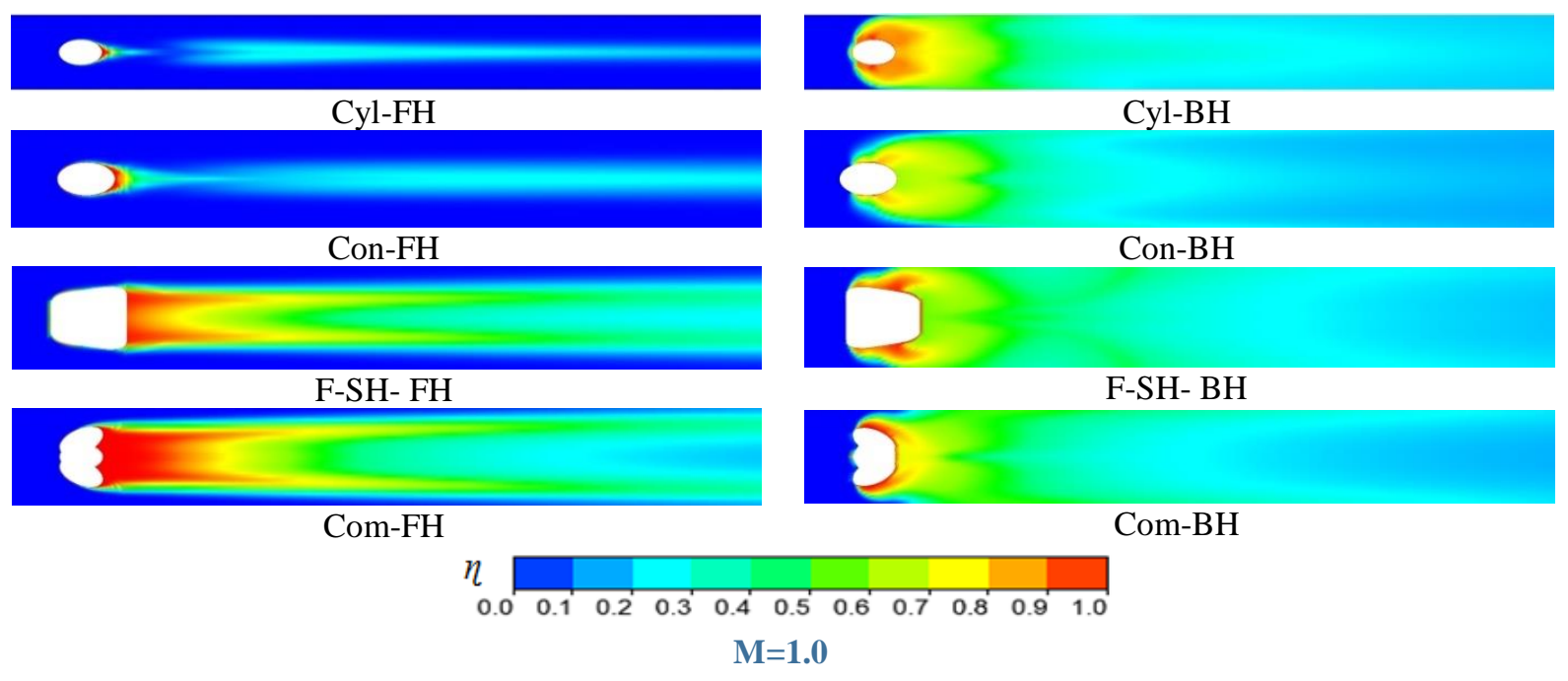

Figure 8. Local film cooling effectiveness distributions
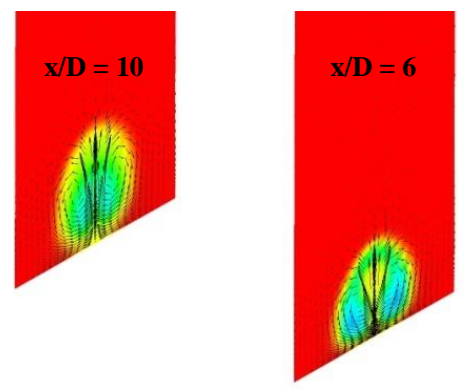

Cyl-FH
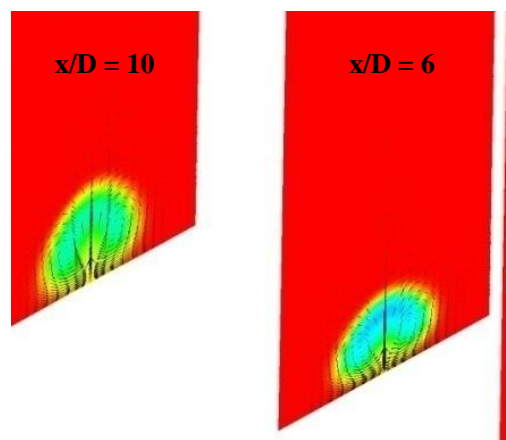

Con-FH

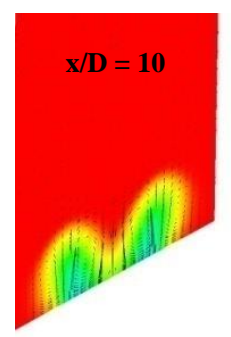

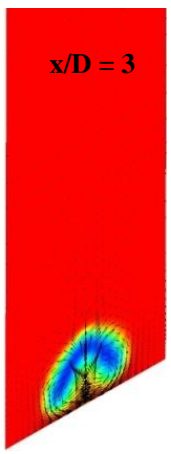
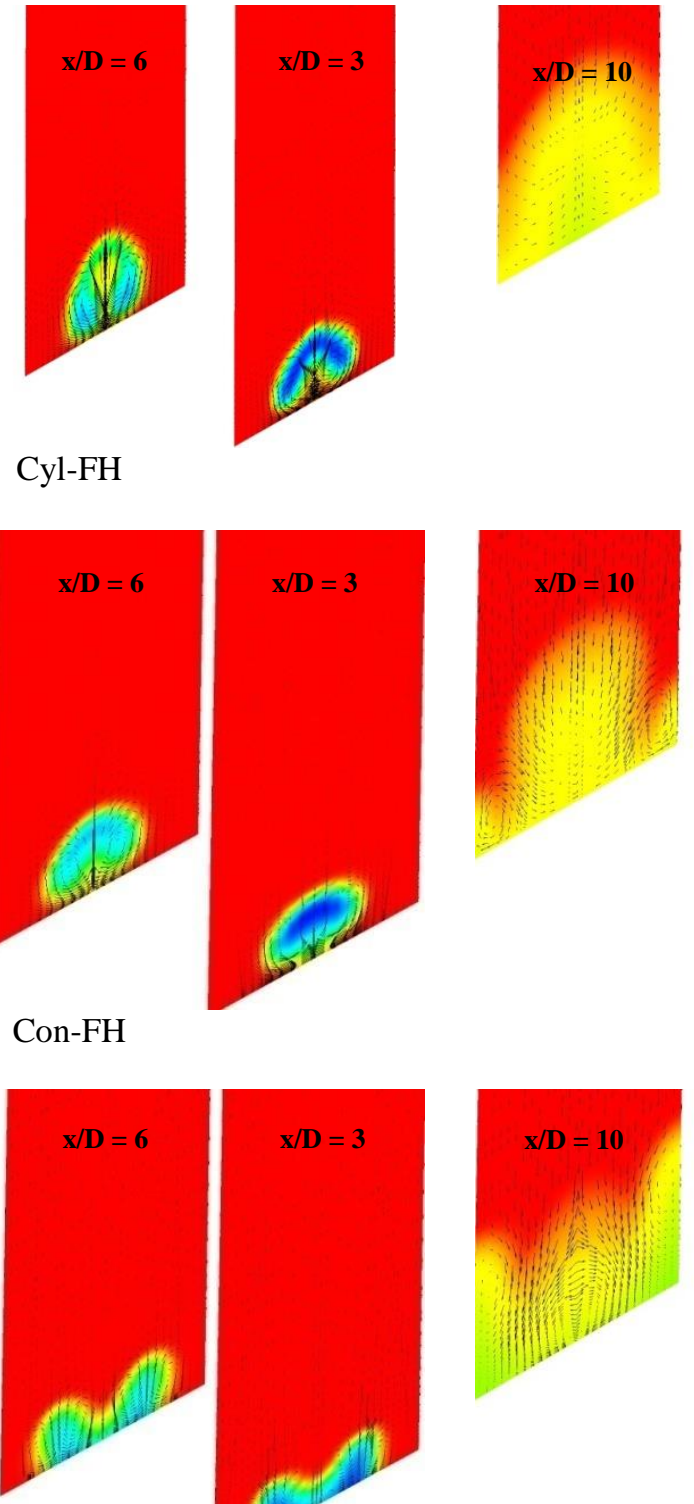

F-SH- FH
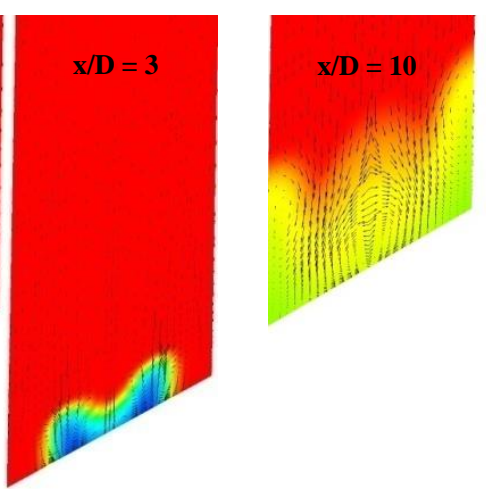

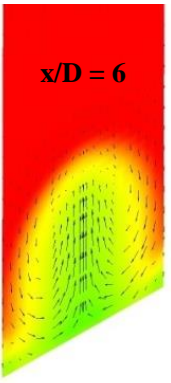

Cyl-BH

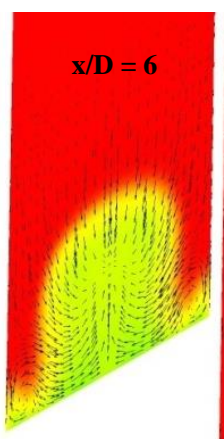

\section{Con-BH}
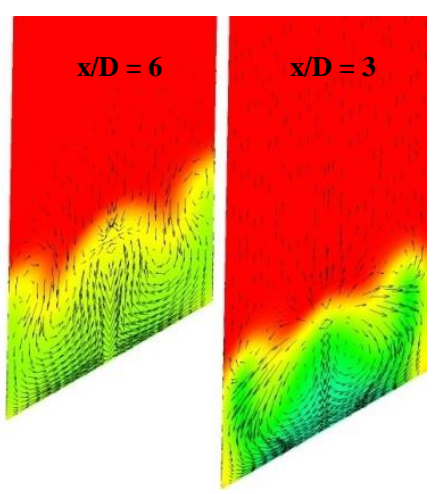

F-SH- BH 


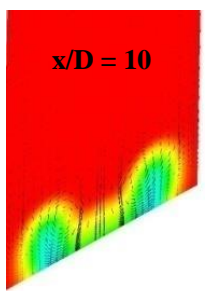

Com-FH
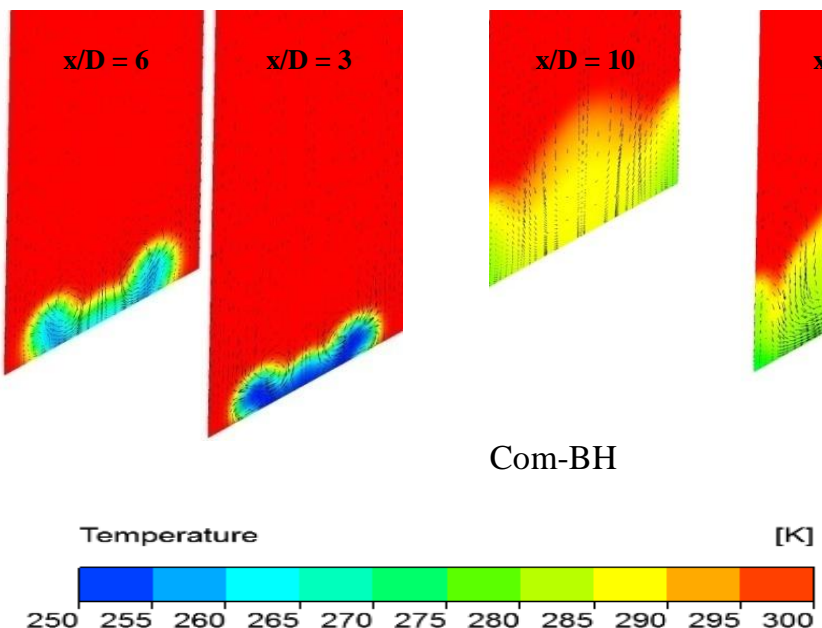
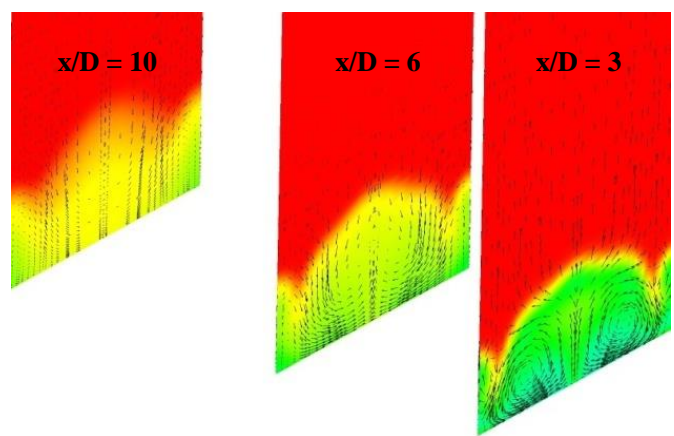

Com-BH

Figure 9. Velocity vectors and temperature contours at $\mathrm{x} / \mathrm{D}=3, \mathrm{x} / \mathrm{D}=6$ and $\mathrm{x} / \mathrm{D}=10$, at $\mathrm{M}=1.0$

\section{CONCLUSIONS}

Film cooling performance of four-hole shapes with the two injections, forward and backward are carried out in the present research. The cooling effectiveness of the eight cases are examined at three blowing ratios, $\mathrm{M}=0.5,1.0$ and 1.5 . From the numerical analysis, the main conclusions are as follows:

1. For the cylindrical hole, the backward injection provides a higher centerline and lateral averaged film cooling effectiveness than the forward injection when $M>0.5$. For the conical hole, a best film cooling effectiveness on the centerline at $\mathrm{M}=0.5$ is obtained with forward injection compared with all other cases, whereas the conical hole increases the velocity of the coolant flow due to its extended angle. While at higher blowing ratio, the improvement of downstream and lateral average effectiveness is shown in backward injection case.

2. For the fan-shaped hole and combined hole, the film cooling effectiveness increases with the increase of blowing ratios for the both injections (forward and backward). a better film cooling effectiveness on the centerline is shown using the forward injection, where the backward injection enhances the lateral average film cooling effectiveness in the case of $M>0.5$.

3. For different shape holes with forward injection, the two rotating vortex pair are much stronger and their size clearly increases as the downstream distance increases. In the backward injection case, these vortexes are less intense and the coolant flow barely detaches from the wall. Thus, the film cooling effectiveness is more uniform with the backward injection hole than that with the forward injection hole improving the film cooling protection.

\section{REFERENCES}

[1] F. Ebacher, "Analyse du refroidissement par film de la paroi de bout de pales d'une turbine en céramique à configuration renversée," M.Sc. Theses, 2017.

[2] R. J. Goldstein, “Film Cooling,” Adv. Heat Transf., vol. 7, no. C, pp. 321-379, 1971, doi: 10.1016/S0065-2717(08)70020-0.

[3] R. J. Goldstein, E. R. G. Eckert, and F. Burggraf, "Effects of hole geometry and density on three-dimensional film cooling," Int. J. Heat Mass Transf., vol. 17, no. 5, pp. 595-607, 1974, doi: 10.1016/0017-9310(74)90007-6.

[4] M. Gritsch, A. Schulz, and S. Wittig, "Adiabatic wall effectiveness measurements of film-cooling holes with expanded exits,” Proc. ASME Turbo Expo, vol. 3, no. July 1998, pp. 549-556, 1997, doi: 10.1115/97-GT-164.

[5] C. Saumweber, A. Schulz, and S. Wittig, "Free-stream turbulence effects on film cooling with shaped holes," J. Turbomach., vol. 125, no. 1, pp. 65-73, 2003, doi: 10.1115/1.1515336.

[6] I. C. Lee, Y. C. Chang, P. P. Ding, and P. H. Chen, "Film cooling over a concave surface through two Staggered rows of compound angle holes," J. Chinese Inst. Eng. Trans. Chinese Inst. Eng. A/Chung-kuo K. Ch'eng Hsuch K'an, vol. 28, no. 5, pp. 827-836, 2005, doi: 10.1080/02533839.2005.9671053.

[7] M. Miao and C. Y. Wu, "Numerical approach to hole shape effect on film cooling effectiveness over flat plate including internal impingement cooling chamber," Int. J. Heat Mass Transf., vol. 49, no. 5-6, pp. 919-938, 2006, doi: 10.1016/j.ijheatmasstransfer.2005.09.015.

[8] A. Azzi and B. A. Jubran, "Numerical modelling of film cooling from converging slot-hole," Heat Mass Transf. und Stoffuebertragung, vol. 43, no. 4, pp. 381-388, 2007, doi: 10.1007/s00231-006-0115-9.

[9] A. Khorsi and A. Azzi, "Computation film cooling from three different holes geometries," Mechanika, vol. 86, no. 6, pp. 3237, 2010, doi: 10.5755/j01.mech.86.6.15971.

[10] G. Li, H. Zhu, and H. Fan, "Influences of hole shape on film cooling characteristics with CO2 injection," Chinese J. Aeronaut., vol. 21, no. 5, pp. 393-401, 2008, doi: 10.1016/S1000-9361(08)60051-5. 
[11] C. liang Liu, H. ren Zhu, J. tao Bai, and D. chun Xu, "Film cooling performance of converging-slot holes with different exitentry area ratios," J. Turbomach., vol. 133, no. 1, pp. 1-11, 2011, doi: 10.1115/1.4000543.

[12] C. Liu, H. Zhu, J. Bai, and D. Xu, "Film Cooling Performance of Waist-Shaped Slot Holes." pp. 1359-1370, Jun. 14, 2010, doi: $10.1115 /$ GT2010-22237.

[13] C. Q. Nguyen, P. L. Johnson, B. C. Bernier, S. H. Ho, and J. S. Kapat, "Comparison of film effectiveness and cooling uniformity of conical and cylindrical-shaped film hole with coolant-exit temperature correction." pp. 1897-1907, Jun. 14, 2010, doi: 10.1115/GT2010-23732.

[14] C. Han, Z. Chi, J. Ren, and H. Jiang, "Optimal arrangement of combined-hole for improving film cooling effectiveness." Turbo Expo.: Power for Land, Sea and Air, 2013, doi: 10.1115/GT2013-94561.

[15] H. Hassan and K. Abdullah, "Combined-hole film cooling with the application of double flow control devices," MATEC Web Conf., vol. 135, pp. 1-9, 2017, doi: 10.1051/matecconf/201713500003.

[16] J. Wang, K. Tian, J. Luo, and B. Sundén, "Effect of hole configurations on film cooling performance," Numer. Heat Transf. Part A Appl., vol. 75, no. 11, pp. 725-738, 2019, doi: 10.1080/10407782.2019.1608762.

[17] S. Na and T. I.-P. Shih, "Increasing adiabatic film-cooling effectiveness by using an upstream ramp." Turbo Expo.: Power for Land, Sea and Air, pp. 931-938, May 08, 2006, doi: 10.1115/GT2006-91163.

[18] K. B. M. Q. Zaman, D. L. Rigby, and J. D. Heidmann, "Experimental study of an inclined jet-in-cross-flow interacting with a vortex generator," 48th AIAA Aerosp. Sci. Meet. Incl. New Horizons Forum Aerosp. Expo., no. January, pp. 1-18, 2010, doi: 10.2514/6.2010-88.

[19] A. F. Shinn and S. Pratap Vanka, "Large eddy simulations of film-cooling flows with a micro-ramp vortex generator," $J$. Turbomach., vol. 135, no. 1, Oct. 2012, doi: 10.1115/1.4006329.

[20] B. An, J. Liu, C. Zhang, and S. Zhou, "Film cooling of cylindrical hole with a downstream short crescent-shaped block," $J$. Heat Transfer, vol. 135, no. 3, Feb. 2013, doi: 10.1115/1.4007879.

[21] C. Zhang and Z. Wang, "Effect of the downstream crescent-shaped block height on the flat-plate film flow and cooling performance," J. Appl. Mech. Tech. Phys., vol. 59, no. 5, pp. 951-961, 2018, doi: 10.1134/S0021894418050255.

[22] W. Zhou and H. Hu, "Improvements of film cooling effectiveness by using Barchan dune shaped ramps," Int. J. Heat Mass Transf., vol. 103, no. I, pp. 443-456, 2016, doi: 10.1016/j.ijheatmasstransfer.2016.07.066.

[23] W. Zhou and H. Hu, "A novel sand-dune-inspired design for improved film cooling performance," Int. J. Heat Mass Transf., vol. 110, pp. 908-920, 2017, doi: 10.1016/j.ijheatmasstransfer.2017.03.091.

[24] X. Li, "Numerical simulation on fluid flow and heat transfer of film cooling with backward injection." $14^{\text {th }}$ Int. Heat Transf. Conf., pp. 257-265, Aug. 08, 2010, doi: 10.1115/IHTC14-22995.

[25] G. Subbuswamy, X. Li, and K. Gharat, "Numerical simulation of backward film cooling with fan-shaped holes." Heat Trans. Summer Conf., Jul. 14, 2013, doi: 10.1115/HT2013-17801.

[26] S. Park, E. Y. Jung, S. H. Kim, H.-S. Sohn, and H. H. Cho, "Enhancement of Film Cooling Effectiveness Using Backward Injection Holes.” Jun. 15, 2015, doi: 10.1115/GT2015-43853.

[27] K. Singh, B. Premachandran, and M. R. Ravi, "Experimental and numerical studies on film cooling with reverse/backward coolant injection," Int. J. Therm. Sci., vol. 111, pp. 390-408, 2017, doi: 10.1016/j.ijthermalsci.2016.09.027.

[28] Z. C. Zhao, L. M. He, S. J. Dai, and S. Shao, "Computational research on film cooling performance of different shaped holes with backward and forward injection," AIP Adv., vol. 9, no. 5, 2019, doi: 10.1063/1.5091573.

[29] A. K. Sinha, D. G. Bogard, and M. E. Crawford, "Film-cooling effectiveness downstream of a single row of holes with variable density ratio," J. Turbomach., vol. 113, no. 3, pp. 442-449, 1991, doi: 10.1115/1.2927894.

[30] V. Yakhot and L. M. Smith, "The renormalization group, the $\varepsilon$-expansion and derivation of turbulence models," J. Sci. Comput., vol. 7, no. 1, pp. 35-61, 1992, doi: 10.1007/BF01060210. 